\title{
Direct time-domain shaping of high-energy femtosecond pulses at THz burst frequencies
}

\author{
Tobias Flöry ${ }^{1}$, Edgar Kaksis ${ }^{1}$, Ignas Astrauskas ${ }^{1}$, Tadas Balčiūnas ${ }^{1}$, Audrius Pugžlys ${ }^{1,2}$, \\ Andrius Baltuška ${ }^{1,2^{*}}$, Daniil Kartashov ${ }^{3}$, Alexander Mitrofanov ${ }^{4}$, Andrey Fedotov ${ }^{4}$, Dmitriy \\ Sidorov-Biryukov ${ }^{4}$, Alexei Zheltikov ${ }^{4,5,6}$ Gergö Krizsán $^{7,8}$, Gyula Polónyi ${ }^{7,8}$, József Fülöp ${ }^{7,8}$ \\ ${ }^{1}$ Photonics Institute, TU Wien, Gusshausstrasse 27-387, A-1040 Vienna, Austria \\ ${ }^{2}$ Center for Physical Sciences \& Technology, Savanoriu Ave. 231 LT-02300 Vilnius, Lithuania \\ ${ }^{3}$ Friedrich-Schiller University Jena, Max-Wien Platz 1, 07743 Jena, Germany \\ ${ }^{4}$ Physics Department, M.V. Lomonosov Moscow State University, 119992 Moscow, Russia \\ ${ }^{5}$ Russian Quantum Center, ul. Novaya 100, Skolkovo, Moscow Region, 143025 Russia \\ ${ }^{6}$ Department of Physics and Astronomy, Texas A\&M, College Station TX, 77843- 4242, USA \\ ${ }^{7}$ MTA-PTE High-Field Terahertz Research Group, 7624 Pécs, Hungary \\ ${ }^{8}$ ELI-ALPS, ELI-Hu Nkft., 6720 Szeged, Hungary
}

\begin{abstract}
We generate fully controllable fs multimillijoule pulse bursts with the energy handling, throughput efficiency and frequency resolution substantially exceeding that achievable in spatial-light-modulator and interferometric techniques. The demonstrated proof-of-concept experiments include coherent control of nitrogen-ion emission via multiple-pulse excitation and generation of tunable narrowband $\mathrm{THz}$ pulses via optical rectification.
\end{abstract}

Generation of deterministic femtosecond pulse bursts with inter-pulse temporal separation ranging from fs to $\mathrm{ns}$ is required in a broad range of applications in spectroscopy [1], strong-field physics [2], laser materials processing [3], free electron laser technology [4], etc. An opportunity to control inter-pulse delay within the excitation pulse train (i.e. control over the = intra-burst frequency) directly in the time domain would enable very attractive types of vibrational and rotational Raman spectroscopies based on a fixed laser spectrum and single beam geometries. Another very promising application field is generation of tunable THz pulses via laser pulse burst rectification [5].

Currently, a technological gap exists for efficient THz burst formation and shaping due to intrinsic limitations of the available techniques. The powerful spatial light modulator (SLM) approach is inadequate for broadband pulse shaping at $\mathrm{THz}$ modulation frequencies due to an impossibly high number of spectral channels needed to satisfy both the pulse bandwidth and spectral resolution requirements. Consequently, the achievable pulse delays, depending on the modulation channel density, rarely exceed several ps for pulses with durations around 100 fs. Tunable etalons permit generation of large flexible delays in a single beam, but cannot yield short pulse trains with equal amplitudes. Finally, beam splitting and recombination with dichroic and/or polarization-sensitive optics permits

*Corresponding author: baltuska@,tuwien.ac.at 
generation of arbitrary pulse sequences with controllable interpulse delays and individual pulse amplitude attenuation. All three approaches entail very hefty throughput losses. Moreover, pulse division technique by definition sacrifices at least $50 \%$ of energy in each step of creating a same-polarized pulse pair and makes it very challenging to ensure perfect spatial overlap of the recombined beams. Consequently, none of the discussed approaches is capable of providing the stability, efficiency and pulse format flexibility required for high-intensity applications such as high-order harmonic generation and femtosecond filamentation in gases. Here, we demonstrate a conceptually different, very robust and flexible approach to generate amplified multimillijoule pulse trains with tunable burst frequencies, pulse quantities and individually adjusted pulse amplitudes and phases.

Figure 1a schematically depicts our setup for pulse burst generation via the Vernier effect by use of an oscillator and a regenerative-amplifier (RA) with two appropriately adjusted cavity roundtrip times. A three-state (off, partially closed and closed, Fig.1b) electro-optic switch (Pockels cell) allows injecting a controllable number of pulses into the RA cavity and then amplifying them. Such pulse burst generation method allows achieving a very high repetition rate burst, many orders of magnitude higher than the fundamentaloscillator- or even harmonic repetition frequency, and permitting generation of tunablefrequency $\mathrm{THz}$ pulses via optical rectification of the RA output. An essential limitation of a direct application of this technique is the problem of optical damage in the laser amplifier associated with the emergence of a $\mathrm{THz}$ comb on the spectrum of the amplified pulse. Because of the frequency-to-time mapping of chirped pulses in a CPA amplifier, which translates spectral modulation into temporal peaks, a sequence of $\mathrm{N}$ compressed pulse replicas would carry the total burst energy of $1 / \mathrm{N}$ and an individual replica energy of only 1/N2 compared to a single pulse amplified in the same CPA below its damage threshold. This problem and its solution are explained in Fig.1c and 1d.

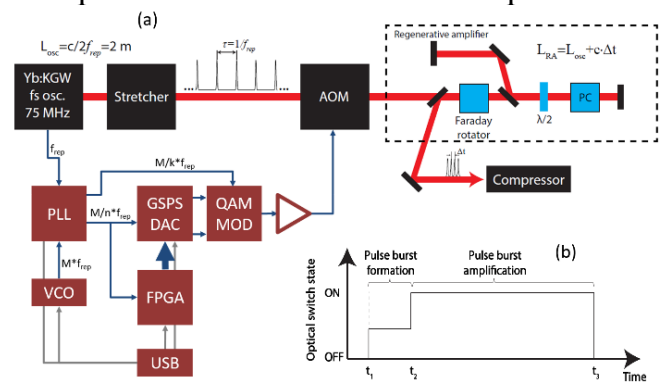

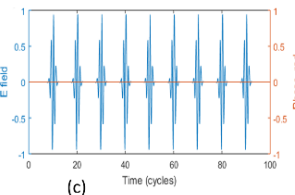

(c)

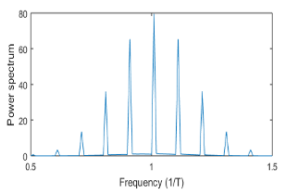

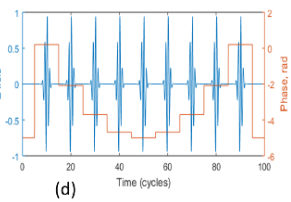

(d)

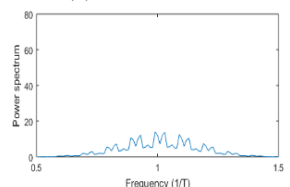

Fig. 1. Experimental scheme for regenerative pulse burst amplification using the Vernier effect. (a) Experimental layout. PC -Pockels cell. (b) Timing diagram of the high-voltage signal on the Pockels cell. (c) Formation of high-intensity spectral modes for bursts with repeated pulse phases. (d) Suppression of spectral modulation via pulse phase encoding by means of AOM.

As shown in Fig.1d, phase randomization of the injected seed pulses becomes necessary to suppress spectral comb formation. By driving the AOM with an amplitude and phase modulated signal (QAM) simultaneous amplitude and phase control of the oscillator pulses is achieved. The QAM signal is generated in a dedicated broadband rf mixer, which is driven by a 4 channel, 16 bit, 1.5 gigasamples per second digital-to-analog converter (DAC). To load data at a sufficient rate (up to $256 \mathrm{GBit} / \mathrm{second}$ ) into the DAC a fast field programmable gate array (FPGA) is used. The AOM carrier frequency, the DAC and FPGA clock are derived from and phase locked to the oscillator frequency, this guarantees stable phase and amplitude control of the oscillator pulses.

As a proof of concept, we generate a $10-\mathrm{mJ}$ burst consisting of 2-10 pulses from a 1030-nm 200-fs $\mathrm{Yb}: \mathrm{CaF}_{2} \mathrm{RA}$ and apply this burst to drive filamentation in nitrogen. Tuning the inter-pulse period (Fig. 2a) around the rotation revival period we confirm the known 
effect of filamentation enhancement (Fig. 2b,c,d) and observe subtle behaviour of laser-like emission of the $\mathrm{N}_{2}{ }^{+}$ion at $391 \mathrm{~nm}$ exactly timed to the full or fractional rotational revival.
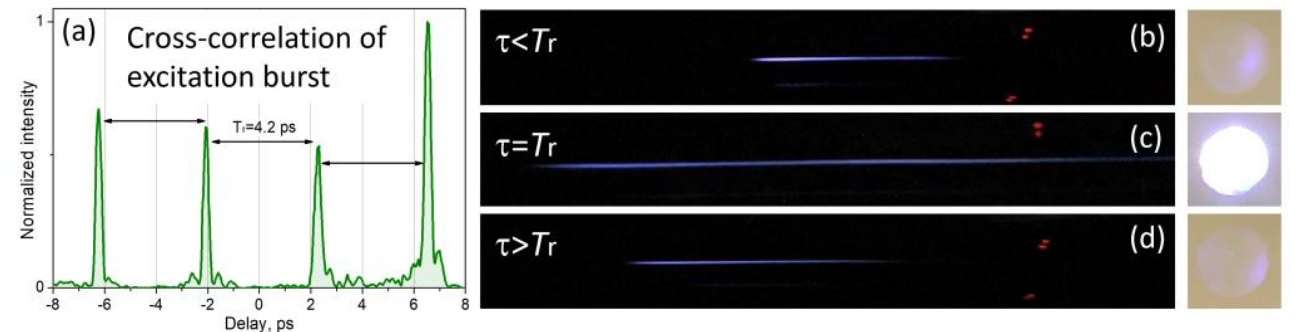

Fig. 2. (a) Measured cross-correlation of a 4-pulse burst tuned to the rotational half-revivals of $\mathrm{N}_{2}$ and programmed to have a high-intensity ionizing pulse following last after a sequence of aligning pulses. (b),(c),(d), Dependence of the $\mathrm{N}_{2}$ plasma channel glow and output beam profile for the 4-pulse burst tuned on resonance (c) and detuned from resonance by $\pm 200 \mathrm{fs}$.

Another proof of concept, demonstrating generation of tunable $\mathrm{THz}$ pulses by optical rectification of an amplified Vernier pulse train, is summarized in Fig. 3. In contrast with the single-pulse rectification resulting in a monocycle interferometric autocorrelation trace and a corresponding broadband spectrum, rectification of a four-pulse train yields tunable narrowband spectra with the center of mass located at the interburst frequency.
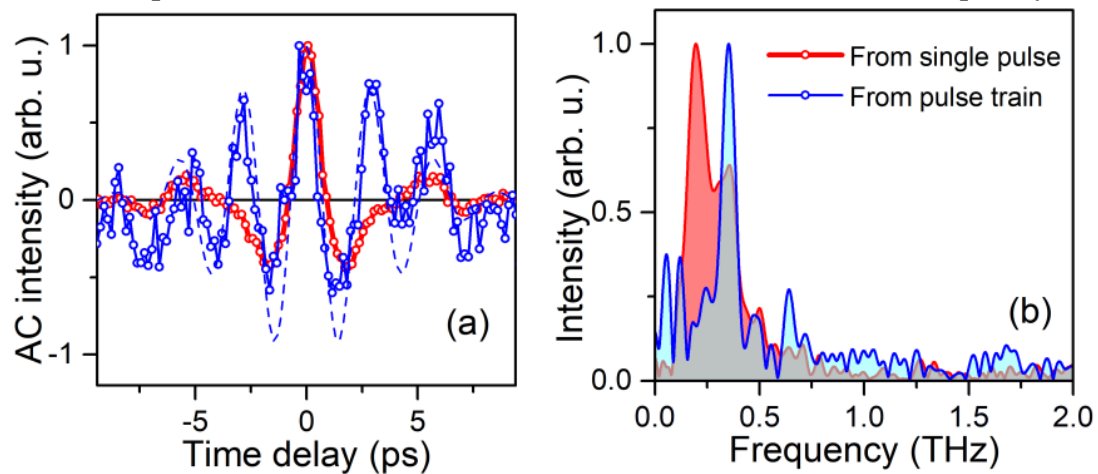

Fig. 3. Autocorrelation traces (a) and Fourier-transform corresponding power spectra (b) for singlepulse and tunable pulse-train rectification in lithium niobate. Note that strong noise on the multicycle autocorrelation is due to absence of relative cavity length stabilization that is yet to be implemented.

\section{References}

1. "Spatially-encoded, single-shot ultrafast spectroscopies," J.T. Fourkas, L. Dhar, K.A. Nelson, and R. Trebino, J. Opt. Soc. Am. B 12, 155-165 (1994).

2. S. Varma, Y.-H. Chen, J. P. Palastro, A. B. Fallahkair, E. W. Rosenthal, T. Antonsen, and H. M. Milchberg, "Molecular quantum wake-induced pulse shaping and extension of femtosecond air filaments", Phys. Rev. A 86, 023850 (2012).

3. C.Kerse, et al., "Ablation-cooled material removal with ultrafast bursts of pulses", Nature 537(7618), 84-88 (2016).

4. M. Kellert, K. Kruse, M. Pergament, G. Kulcsar, T. Mans, and M. Lederer, in CLEO EUROPE/IQEC 2013, pp. 1-1 (IEEE, 2013).

5. Messner, C., Sailer, M., Kostner, H. et al. "Coherent generation of tunable, narrowband $\mathrm{THz}$ radiation by optical rectification of femtosecond pulse trains", Appl Phys B (1997). 